\title{
The role of metrology in the field of medical devices
}

\author{
M. do Céu Ferreira* \\ Portuguese Institute for Quality, Unit of Legal Metrology, Rua C. à Av. dos três Vales, 2825 Monte de Caparica, Portugal
}

Received: 26 April 2011 / Accepted: 10 August 2011

\begin{abstract}
Medical measurements are present in the everyday life of people and are fundamental processes in prevention, diagnosis and treatment of disease. Therefore, a growing interest lies in the role of metrological decisions and conformity assessment, namely where measurements are in support of health. This paper focuses on the use of medical devices looking to enhance its metrological traceability, highlighting the specific role of metrology in the field of health care and the impact of legal control in the framework of the medical devices regulation with a measuring function. It is proposed a new regulatory approach for medical devices in use, facing the convergence between European policy enforcement and Metrological regulations.
\end{abstract}

Keywords: Metrology; traceability; medical measurement

\section{Introduction}

The science of measurement, as a field of technical and scientific activity, has a range of activities with a key role in all sectors of society.

In the health sector, due to the inherent risk of life it is necessary to measure quantities as accurate as possible. Such measurements and measuring instruments are present in the everyday life of people and are fundamental processes in prevention, diagnosis and treatment of disease. Although medical measurements (measurements being relevant to medical diagnosis) are only pieces within the complex process of medical decision making in general, they contribute incrementally. Therefore, the accuracy and reliability of medical measurements are of direct consequences for the health of each individual patient.

Usually, medical decisions are often based on statistical analysis and conclusions of clinical studies [1]. Medical measurements are incorporated within these studies and correlated with other medical findings. Consequently, each medical decision for an individual may be influenced by the results of previous studies, including the data from medical measurements.

Assuming that medical measurements are related with S.I. (International System of Units) quantities, such as mass, temperature, length, etc, it seems that the main concepts of other areas in industrial and legal metrology, can be adapted and used more or less straight forward. Following this line of reasoning, the need to improve the metrological traceability of measuring instruments to make them suitable for use (reproducibility and repeatability conditions), is obvious. Hence, quality assurance of measurement should be ensured by metrological tools, e.g., calibrations, legal metrological control and reference

^ Correspondence: mcferreira@mail.ipq.pt measurement methods. Considering the analogy between other sectors of society, in which the practice of legal metrological control is required, the accuracy of measurements and the traceability chain are essential for the reliability of results.

\section{Regulatory domains for medical devices}

The economic integration of the European Union (EU) has imposed that the EU directives passed to define essential requirements for health, safety, and welfare, with the gradual elimination of barriers to trade among member countries. This methodology deals with the harmonization of Member States legislation, contributing for the single market developing and assurance the free circulation.

Indeed, this scenario has been achieved by the European Council resolution adopted in May 1985, which became known as the New Approach. Therefore, under the scope of the New Approach directives, it was removed technical barriers to trade, promoting the trust to economic operators allowing for the free movement of goods inside the EU.

The regulatory framework for medical devices consists of 3 main directives:

- Directive 90/385/EEC regarding active implantable medical devices;

- Directive 93/42/EEC regarding medical devices;

- Directive 98/79/EEC regarding in vitro diagnostic medical devices [2].

These European directives improve new responsibilities and technical requirements related to instrument's design and manufacturing, before placing them in the market, where each participant (manufacturer, distributer/importer, user, public and government) has an 
important role. Although a wide range of instruments are covered by medical devices directive, this sphere of regulation allows each Member State to consider additional measures to protect public health and citizens. In fact, after placing them in the market and putting them into service no further regulated control (according EU policy) exists in those medical devices.

Products that fall within their scope must meet all applicable essential requirements and must be CE marked to show that they comply. Such products may then be freely sold throughout the European Economic Area (EEA) without being the subject of additional national legislation, except in the field of funding and reimbursement [3].

According international needs, the 3 main directives have been supplemented over time by several modifying and implementing directives, including the last technical revision brought about by Directive 2007/47 EEC [4] which was transpose for Portuguese legislation by LawDecree No. 145/2009, 17 June.

\subsection{Medical devices terms harmonization}

The term medical device includes everything from highly sophisticated computerized medical equipment down to simple wooden tongue depressors, including a wide range of products varying in complexity and application. Several different international classification systems for medical devices are still in use in the world today [5]. However, considering it's relevance in patient safety it is very important to achieve harmonization in medical device nomenclature.

According the Directive 2007/47/EC, medical device means any instrument, apparatus, appliance, software, material or other article, whether used alone or in combination, together with any accessories, including the software intended by its manufacturer to be used specifically for diagnostic and/or therapeutic purposes and necessary for its proper application, intended by the manufacturer to be used for human beings for the purpose of:

- diagnosis, prevention, monitoring, treatment or alleviation of disease;

- diagnosis, monitoring, treatment, alleviation of or compensation for an injury or handicap;

- investigation, replacement or modification of the anatomy or of a physiological process;

- control of conception;

and which does not achieve its principal intended action in or on the human body by pharmacological, immunological or metabolic means, but which may be assisted in its function by such means.

\subsection{The impact of medical devices in the world economy}

In the health sector, medical technology offers a wide range of products covered by community legislation and consequent transposed into national law. This sector has a
Table 1. European Medical Technology Employment. Source: Eucomed, 'An introduction to the Medical Technology industry' [6].

\begin{tabular}{lrr}
\hline Country & No. of Employees & \% of European \\
\hline Austria & 6000 & 1.4 \\
Belgium & 5500 & 1.3 \\
Czech Republic & 12760 & 2.9 \\
Denmark & 14000 & 3.2 \\
Finland & 3000 & 0.7 \\
France & 40000 & 9.2 \\
Germany & 110000 & 25.3 \\
Greece & 2500 & 0.6 \\
Hungary & 4250 & 1.0 \\
Ireland & 26000 & 6.0 \\
Italy & 29815 & 6.9 \\
Netherlands & 9500 & 2.2 \\
Norway & 500 & 0.1 \\
Poland & 8700 & 2.0 \\
Portugal & 3200 & 0.7 \\
Romania & 15000 & 3.5 \\
Slovakia & 2198 & 0.5 \\
Slovenia & 1237 & 0.3 \\
Spain & 25400 & 5.8 \\
Sweden & 15000 & 3.5 \\
Switzerland & 40000 & 9.2 \\
United Kingdom & 60000 & 13.8 \\
\hline Total Europe & 434560 & $100 \%$ \\
\hline
\end{tabular}

dynamic and competitive industry within a global market nearly 10000 types of products.

According Eucomed database [6], in 2007 the community market amounts to $€ 72$ billion with an increasing trend (annual growth up to 6\%). It is the second biggest market worldwide $( \pm 33 \%)$ after the US $( \pm 37 \%)$ and before Japan $( \pm 15 \%)$. The medical device industry employs more than 500000 people in Europe.

Table 1 show the number of employees in European Zone 2007.

In Portugal, the medical technology industry has a low impact on the trade balance.

\subsection{The live span of a medical device}

The principal phases in the life span of a medical device include the conceptions and development planning, manufacturing, packaging and labeling, advertising, sale and use. Any of these may overlap and interactions can affect the safety and performance of a medical device.

Usually, the manufacturer is engaged with the first three phases of the medical device's life span and the user is a professional in a health care facility, but may also be the patients. The manufacturer is responsible for ensuring that products conform to applicable legal requirements. It plays an important role to: (a) produce the device and (b) represents the principal entity (technology/industry sector) involved in the pre-marketing device. The notified body is another actor with an important responsible for carrying out tasks relating to the procedures conformity 
assessment. This is a competent authority appoint by the government of each Member State with authority to act on behalf of the government in order to ensure that the requirements of the medical device directives are transposed into National law and are applied.

\subsubsection{Registration, control and supervision of a medical device}

Once you make the assessment of medical devices compliance with requirements and affixing the CE marking, they can be traded freely. However, several Member States, including Portugal, require a registration system for products, so that they can control the outstanding products in their market.

Facing the actual situation, in the framework of the EC Member States are concerned with the same European regulates until placing on the market and putting them into service.

Member States are free, under the management of their health systems of share to a varying medical device that deliver benefits to understand short, medium or long term care to their patients.

In a global market with so many outstanding products and so diverse, associated to a limited human resources by the authorities, more and more Member States set approaches for the users of these products in order to detect suspicious errors or failures of the market. A surveillance system for the in use instruments is a requirement for all medical devices, leaving the manufacturer to identify all of the events likely to cause serious injury or death to a patient, a user or a third party [7].

Due to this knowledge, the politics of medicine, medical devices and health technology assessment manufacturer shall submit a report on its occurrence to the competent authority of that country, in order to centrally analysis and register it and consequently, trigger the appropriate measures to eliminate or minimize the possibility of recurrence of the event. Regarding the undeniable importance of this approach, the impact of the metrological traceability of the medical devices with measuring functions is strongly important and must be highlighted by the competent authorities. However, in the current condition, it seems that this matter has been left out of interest.

All medical devices must satisfy the systems of premarket review, such as risk management for the requirements of safety and performance, quality system (some low-risk devices may be exempt) and labeling. However, the degree of regulatory scrutiny increases with the potential risks of the medical device, as evidenced by the risk-based device classification system [8].

In Portugal, the regulatory surveillance system for medical devices in use is still far away (and unknown) of its major audience (users and health professionals). Any strategy for future development in this field has to pass through an increased participation of users in the system, which includes the governmental cooperation by a closer relationship between involved ministries and organizations allowing to bring benefit for the entire system of health and economics. Regarding the fact that better measurements lead to reduce costs, an interdisciplinary task force must be improved with a transversal application for the field of medical devices.

\section{Considerations of metrological assurance of medical measurements}

The science of measurements is part of a technical community as a whole, providing tools to ensure the reliability of the technologies used. To the common user, these metrology tools can either be relevant or irrelevant, but for life safety, the improvement given by metrology is present every day and everywhere.

Measurements are essential in medical diagnosis and the prevention and treatment of diseases, risk assessment and monitoring of patients. Such measurements performed with measuring instruments are present in the everyday life of people. Moreover, as the measurement results become more important in medicine they should be accurate, actual and comparable in different locations over time. Only then, it will be possible optimize patient care and efficient management of funds applied to health care.

In the Portuguese health sector, has verified the increasing of the implementation of a set of initiatives aimed to assurance, such as certification/accreditation of the public and private hospitals. In this context, the use of medical devices with measuring function should not pass outside the qualification process. However, at the present time in some situations, the standards requirement for the metrological traceability is neglected. Thus, as a part of accredited/certified activities, the measuring instruments must demonstrate compliance with metrological traceability chain through the evidence of their certificates of calibration/verification on the basis of voluntary or policy regulation. These certificates will be valid if issued by a laboratory accredited for the activities concerned, or by a national metrology laboratory (both assigned the mutual recognition agreement).

In the aim of metrology, trust means measurements traceability to SI with a certain confidence level. In the health sector, by the inherent risk of life it is necessary to measure quantities as accurate as possible [9].

A simple example is the incorrect blood pressure that is a major risk for health (in some particularly disease): this example presents a measurement system marked by a technology, where the metrological control has a key role through technical proceedings.

In order to demonstrate the impact of traceability of medical equipment in public health, several studies have been conducted focusing the accuracy of measurements and the traceability chain as an essential tool for the guarantee of results. As a results, the findings are worthy of reflection and disclosure [10-15]. On this scope, the World Health Organization (WHO) has published information by promoting good practices for the use of medical equipment. Considering the analogy between other sectors of society, in which the practice of metrological traceability 
Table 2. Recomendation OIML and European Standards for some categories of medical device.

\begin{tabular}{|c|c|c|}
\hline Measuring instrument & OIML & \multirow{3}{*}{$\begin{array}{c}\text { EN } \\
\text { EN1060-1:1995+A2:2009 } \\
\text { EN 1060-2:1995+A1:2009 } \\
\text { EN 1060-3:1997+A2:2009 } \\
\text { EN 1060-4:2004 }\end{array}$} \\
\hline $\begin{array}{l}\text { Mechanical non-invasive } \\
\text { sphygmomanometers }\end{array}$ & R 16-1 (2002) & \\
\hline $\begin{array}{l}\text { Non-invasive automated } \\
\text { sphygmomanometers }\end{array}$ & R16-2 (2002) & \\
\hline Medical syringes & R $26(1978)$ & EN 20594-1:1993 \\
\hline $\begin{array}{l}\text { Standard graduated pipettes for } \\
\text { verification officers }\end{array}$ & R 40 (1981) & EN ISO 4787:2010 \\
\hline $\begin{array}{l}\text { Electroencephalographs - Metrological } \\
\text { characteristics - Methods and equipment for } \\
\text { verification }\end{array}$ & R 89 (1990) & EN 60601-2-26:2003 \\
\hline $\begin{array}{l}\text { Electrocardiographs - Metrological } \\
\text { characteristics - Methods and equipment for } \\
\text { verification }\end{array}$ & R 90 (1990) & $\begin{array}{c}\text { EN 60601-2-25:1995/A1:1999 } \\
\text { EN 60601-2-27:2006 }\end{array}$ \\
\hline $\begin{array}{l}\text { Measuring instrumentation for human } \\
\text { response to vibration }\end{array}$ & R 103 (1992) & EN ISO 8041:2005 \\
\hline $\begin{array}{l}\text { Pure-tone audiometers (including } \\
\text { Annexes A to E) }\end{array}$ & R 104 (1993) & $\begin{array}{l}\text { EN 60645-2:1997 } \\
\text { EN 60645-1:2001 }\end{array}$ \\
\hline $\begin{array}{l}\text { Clinical electrical thermometers for } \\
\text { continuous measurement }\end{array}$ & R 114 (1995) & \multirow[t]{2}{*}{ EN 12470-1,2,3,4:2000 + A1:2010 } \\
\hline $\begin{array}{l}\text { Clinical electrical thermometers with } \\
\text { maximum device }\end{array}$ & R 115 (1995) & \\
\hline Equipment for speech audiometry & R $122(1996)$ & EN 60645-2:1997 \\
\hline
\end{tabular}

is supposed to be regularly enforced, also the health and medicine sphere should not continue to be isolated from the legal metrological checks.

\subsection{The role of standards and recommendations to improve new enforcement}

For some kind of medical devices, several countries have adapted the recommendations of the International Organization of Legal Metrology (OIML), and developed their own regulations. This regulation requires the implementation of legal metrological control, but it can also be viewed as a voluntary act, in the case of calibrations.

According to recommendations of the European Community, all medical devices must meet the requirements of ISO and IEC (International Electrotechnical Committee). In addition, there are also EN (European Normalization) and IEC standards specially adapted to European Community requirements [16]. These standards are developed and harmonized by the European Committee for Normalization (CEN) and European Committee for Electrotechnical Normalization (CENELEC).

Classification of medical devices by potential risk and methods of risk analysis are a substantial step toward convergence between European policy regulates and Metrological National Institutes. Combined application of medical safety by measuring standards and reference standards reduces the potential hazard to patient and medical personnel to a minimum. Therefore, standardization should include requirements for technical service and calibration of medical devices, software control, etc.

On the other hand, there is also Directive 93/42/EEC, 10th item, Annex I related to the requirements of the devices, states that those with a measuring function must be designed to ensure the measurement accuracy. Highlight this requirement is referred throughout the Directive text in different attachments and items ${ }^{1}$. Moreover, as part of quality assurance, this directive is quite demanding with the ISO 13485 [17] as the reference standard for the regulatory requirements. However, 8th section of this standard introduced the requirements for measurement systems which are undemanding. Through its supplement, the reference standard ISO/TR 14969 [18] deepens the measurement subject, which represents as an important input on that. In order to enforce this approach, Table 2 shows the relation between the recommendations from OIML and the corresponded standard reference from EN.

\subsubsection{A new approach for medical measurements regulates}

International Organizations such as WHO and the European Commission itself, identify in many areas of health, medical technology and medical devices as a key input to the collective health through its contribution on reducing disease and improving health patients [19]. Simultaneously, training of health professionals has become an essential point.

However, as a fundamental pillar of this issue, is the consolidation of a metrological knowledge that should be a permanent organization's strategy. The development of this culture and consequent applicability on safety and

\footnotetext{
1 http://ec.europa.eu/enterprise/ policies/european-standards/documents/ harmonised-standards-legislation/list-references/ medical-devices/index_en.htm\#linkBoxTools
} 
instruments performance require long-lasting actions with a widespread of monitoring. Probably, these aspects of cultural dissemination are as important as the concern about technical and legal regulate framework.

According to the group of risk, medical devices fall into four groups [20]: group I requires declaration of correspondence; group IIa requires declaration of correspondence and certification of quality system; group IIb requires declaration of correspondence and independent testing and certification of the quality system; group III requires independent assessment of design and declaration of correspondence and independent testing and certification of the quality system.

These modules have some comparability with the Directive on Measuring Instrument 2004/22/EC (MID). As we know, the philosophy of MID was to adopt a decisively modern regulatory approach, leaving much more room for technological innovation and more choice for manufacturers in conformity assessment procedures, aligning Community legislation on international standards. The objective was to allow free movement of goods between the Member States, while allowing for the possibility of continuing manufacturing in conformity with national rules for the home market. This is the same philosophy of medical devices directive. However, is missing the principle of the metrological traceability chain, which means the aim of this reflection paper This request may be achieved by a new approach, where a convergence for MID structure could be improving a new dynamic and trustful system for the provision of reliable measurements. Indeed, this new approach will be covers the specific metrological requirements for the instruments that are in use.

Each Member State could be improve National legislation in order to apply the specify requirements for the instruments that are placing on the market and putting on use. The applicable metrological requirements must be met and if the instruments are in use for some time they are subject to a re-verification test. The instruments will be rejected after it is demonstrated that it is operating outside its metrological limits (maximum permissible errors). Taking into account the specific national operating conditions, Member Sates should improve a generic network to support and harmonize the metrological limits for each group of medical devices, as a holistic approach. The role of metrology in this field must be also highlighted by new International Standards, in order to improve the definition of metrological requirements, focusing some kind of medical devices with a measuring function. In this context, the verification requirements could be drawn for each type of instrument of an enforcement system and based on specific national regulates. Special attention must be given to all systems including software, to ensure the integrity, authenticity and privacy of the data.

Actually some countries have successfully implemented metrological verification for several categories of medical devices. In the legal framework of Portuguese legislation, some measuring instruments used in the field of X-ray are submitted to a metrological control, according to national regulation No. 1106/2009, 24 September.

\section{Conclusions}

This paper presented a brief overview on the key role of metrology and legal control in the filed of medical measurements, and also some suggestions for new approach focusing the present National and European regulatory system. Taking into account the technological innovation, economic significance and technical barrier, it was given an explanation about the legal framework of Member States and the consequent impact in the field of metrological regulates. It was also highlighted the actual situation in Portugal, where after placed in the market and put into service no further regulated metrological control exists for the major of medical devices with measuring functions. Undoubtedly, this issue plays an important role in field of medicine measurements.

Acknowledgements. The author thanks Eng. Cartaxo Reis (director of legal metrology unit of IPQ) for his contribution to the revision paper.

\section{References}

1. R.C. Brownson, E.A. Baker, T.L. Leet, N. Gillespie, Evidence-Based Public Health (Oxford University Press, New York, 2002)

2. Council directive 98/78/EEC of 27 October 1998 concerning in vitro diagnostic medical devices, Official Journal L 33, $7 / 12 / 1998$

3. S. Jonas, B. Michael, B. Reinhard, Balancing adoption and affordability of medical devices in Europe, Health Policy 92, 218-224 (2009)

4. Council directive 2007/47/EEC of 5 September 2007, Official Journal L 247, 21/09/2007, pp. 0021-0055

5. World Health Organization, Medical Device Regulations (WHO, Geneva, 2003), ISBN 9241546182, http://www. who.int/medical_devices/en/

6. http://www.eucomed.org/press/ /media/ A4261DDBBA854C7F852CA16F7AC3C716.ashx (accessed 2011-01-18)

7. M.P. Keshia, D. Morhaimb, M.A. Williams, The public's perspectives on advance directives: Implications for state legislative and regulatory policy, Health Policy 94, 57-63 (2010)

8. V.P. Gundarov, G.I. Kavalerov, Standardization and Certification of Quality of Medical Devices, Biomed. Eng. 35, 123-126 (2001)

9. Chião et al., Metrology and Standards Needs for Some Categories of Medical Device, J. Res. Natl. Inst. Stand. Technol. 113, 121-129 (2008)

10. M.V. Lucatelli et al., Engenharia clínica e a metrologia em equipamentos eletromédicos, in metrologia para a Vida, Sociedade Brasileira de Metrologia (SBM) (Recife, Pernambuco, Brasil, 2003)

11. G.M. Minyailik, Standardization and certification of medical devices: results and prospects, Biomed. Eng. 35, 121122 (2001); Translated from Meditsinskaya Tekhnika 35, $3-4(2001)$ 
12. E.C. Monteiro, M.L. Lessa, A metrologia na área de saúde: garantia da segurança e da qualidade dos equipamentos electromédicos, Engevista 7, 51-60 (2005)

13. R.D. Sriram, S.J. Fenves, Manufacturing Metrology and standards for the health care enterprise program summary (National Institute of Standards and Technology, 2008), NISTIR 7487

14. M.J. Turner, P.C. Kam, A.B. Baker, Metrology in Medicine, Department of Anesthetics, University of Sydney, 2004, http://www.personal.usyd.edu.au/ $\sim$ mjturner/metrology_in_med_msa_2004.pdf

15. Council directive 90/385/EEC of 20 June 1990 concerning the approximation of the laws of the member states relating to active implantable medical Devices, Official Journal L 189, 20.7.1990, p. 17
16. R. Wilson et al., An analysis of the causes of adverse events from the quality in Australian health care study, Med. J. Aust. 170, 411-415 (1999)

17. Council Directive 93/42/EEC of 14 June 1993 concerning medical devices, Official Journal L 169, 12/07/1993, pp. 0001-004393/42/CE

18. ISO 13485:2003, Medical devices-Quality management systems-requirements for regulatory purposes

19. ISO/TR 14969:2004, Medical devices-Quality management systems- guidance on the application of ISO 13485:2003

20. Report on the functioning of medical devices directive, http://www.bsigroup.com/upload/ Standards\%20 \&\&20Publications/Healthcare/ MDDReport2002.pdf (accessed 2011-01-18) 